of the fundamental problems of the field: which observations are polluted by instrumental noise, and which are genuine deterministic chaos? And how do we know?

Despite my disappointment on this score, I can enthusiastically recommend the book to chemists who want to learn what all the fuss over chemical chaos is about. It is a no-nonsense book, written by a chemist for chemists, without unnecessary mathematical frills or philosophical fancies. The book contains not one colour figure, which I take as a good sign: it is meant not for the coffee table but for serious study by the next generation of chemical kineticists.

John J. Tyson is in the Department of Biology, Virginia Polytechnic Institute and State University, Blacksburg, Virginia 24061, USA.

\section{Mesmer again?}

\section{Cyril W Smith}

Biomagnetism. By Romuald S. Wadas. Ellis Horwood: 1991. Pp. 170. £40.00, $\$ 68$.

IN 1987, the New York State Power Lines Project realized that "the . . . variety of effects of magnetic fields have not been previously appreciated". The whole area of biomagnetism continues to be fraught with controversy and partisan influence. As Romuald Wadas remarks:

Thousands of experiments have been carried out to date, and different conclusions reached therefrom, sometimes contradicting each other. A physical explanation of these effects encountered enormous difficulties. Until recent years, the possibility of a magnetic field having an influence on chemical reactions was altogether denied ... The existence of such effects was looked upon as pure fantasy.

This book is a revised and enlarged translation from the Polish edition, Biomagnetyzm, published in 1978. The translation editor, Professor R. Pethig of University College of North Wales has ensured a well-prepared English text. The book is addressed to all those interested in the physics of magnetic phenomena in biology and claims to be the first book on biomagnetism available in English. It covers the fundamentals of magnetic phenomena together with magnetic molecular complexes, diamagnetic, paramagnetic and ferromagnetic biological molecules, magneto-optical phenomena and the effects of magnetic fields on living organisms through results of selected biological experiments.

What Wadas does well is to cover the physics of magnetic phenomena in a way more likely to be understood by the medical and biological reader, and to spell out the implicit biochemistry for the benefit of the physicist. He explains that

[in] the course of a chemical reaction, when a molecule breaks down but its components have not yet had time to produce new diamagnetic molecules, there exist energy conditions for spin reversal by a magnetic field .... The arrangement of molecule components passes from a singlet-excited to a triplet-excited state.

Thus can magnetic fields, including those generated by paramagnetic molecules and free radicals (paramagnetic on account of their uncompensated spins) and even nuclear magnetic moments, affect the course of chemical reactions.

Wadas describes how liquid crystal molecules, widespread in biological systems, can be structurally deformed by electric and magnetic fields to produce changes in optical activity and membrane properties. He gives a simplified classical description of magnetic resonance and explains what can be derived from measurements of resonance lines. He notes that with a stationary magnetic field of the order of 100 milliteslas in strength, there can be strong influences, both adverse and favourable, on the activity of biological molecules as well as on the blood system, cellular membranes, the operation of the heart, neoplastic cells, enzyme activity, and on the germination and development of plants.

The effects of Earth-strength (50 microteslas) magnetic fields are considered in relation to magnetic compasses in living organisms and radiaesthesia. Wadas quotes from Y. Rocard's chapter in M. F. Barnothy's book (Biological Effects of Magnetic Fields, Plenum, 1964) on the biological effects of magnetic fields, mentioning the threshold of 30 nanoteslas per second for dowsing a magnetic-field gradient and the 2-5-microtesla saturation level, 'windows' that may represent bands within which some form of long-range coherence is established.

Four-fifths of the references date from the 1978 Polish edition, which further restricts the value of a book already priced beyond the reach of most graduate students. The substantial literature following the publication of P. Semm, T. Schneide and L. Vollrath's report in Nature on "Effects of an Earth-strength magnetic field on electrical activity of pineal cells" $(288,607 ; 1980)$ has not been noted; neither has the work of $\mathrm{H}$. Fröhlich on coherence in living systems.

Judging by the programme list for a forthcoming international congress, the book is a decade too late to make an impact among researchers in biomagnetics, but still a decade too early for others to appreciate the significance of the subject. $\square$

Cyril Smith is in the Department of Electronic and Electrical Engineering, University of Salford, Salford M5 $4 W T$, UK.

\section{The Universe defined}

\author{
R. J. Tayler
}

The Astronomy and Astrophysics Encyclopedia. Edited by Stephen P. Maran. Cambridge University Press: 1992. Pp. 1002. £60.

A Concise Dictionary of Astronomy. By Jacqueline Mitton. Oxford University Press: 1991. Pp. 423. £12.95, \$19.95.

THE briefest of perusals of these two reference books suffices to show just how dramatically our knowledge of the Universe has increased in the past 50 years. In 1940, observational astronomy meant optical astronomy, whereas now the Universe is studied in all regions of the electromagnetic spectrum. Larger optical telescopes and more efficient detectors, many carried by satellites, have allowed astronomers to probe deeper into space and hence further back in time. Many new types of objects, such as pulsars, binary X-ray sources, infrared protostars, quasars and radio galaxies, are now known; and, through the discovery of the cosmic microwave radiation, the study of the very early Universe has been made respectable. Even the Solar System is seen in a different light. Space missions have provided detailed information about the atmospheres and surfaces of the planets, led to the discovery of new satellites and allowed detailed measurements of the properties of the Earth's local environment.

The Universe now provides a physical laboratory in which the laws of physics can be tested in a way that is not possible on Earth. White dwarfs once provided a test of quantum mechanical predictions of the properties of a dense degenerate electron gas. Now neutron stars give information about bulk matter at and above nuclear densities; Einstein's general theory of relativity has been given strong support by the discovery of gravitationally lensed quasars and the properties of the binary pulsar; and plasma physics and magnetohydrodynamics are new branches of physics that can be studied particularly well in the interaction of the solar wind and Earth's magnetosphere. Particle physicists have realized that they can never build accelerators to compete with conditions in the very early Universe, and the union of particle physics and cosmology is one of the active fields of current research. But particle physicists in a sense have had their revenge, for it now seems that most of the matter in the Universe is nonluminous, consisting of weakly interacting elementary particles rather than ordinary matter. Even chemistry 